\title{
4 Sachkommentar
}

I-5 Inscriptio

Trotz des stark fragmentierten Zustands der oberen Partie der Inschrift ist die Gestaltung der inscriptio klar erkennbar. Die erste Zeile mit der Nennung der siebenten ludi saeculares ist mit den größten Buchstaben geschrieben; im Folgenden wird die Schriftgröße von Zeile zu Zeile kleiner. Septimius Severus hat sich hier als Kaiser zwar nicht mit der vollständigen Titulatur und Filiation nennen lassen, doch seine wichtigsten Funktionen gestalterisch besonders hervorgehoben. Z. 2 nennt ihn mit den cognomina und Titeln seiner Siege, die seine erfolgreiche Eroberungspolitik bezeugen, Z. 3 gibt seiner tribunicia potestas durch die Position in der Mitte der Zeile mit umgebenden vacat ein besonderes Gewicht. Beide Zeilen sind in fast gleich großen Buchstaben wie Z. I gestaltet. Die besondere Hervorhebung der tribunicia potestas ist für die Durchführung der Säkularspiele wichtig. Auch das augusteische commentarium verzeichnet, dass Augustus und Agrippa die Säkularspiele aufgrund ihrer tribunicia potestas veranstalten konnten und nicht aufgrund anderer Äm-

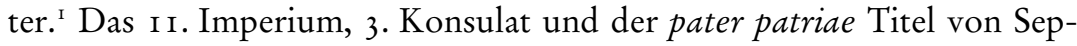
timius Severus sind demzufolge hier nicht erwähnt.

In Z. 4 folgen seine Söhne. Zuerst der ältere Caracalla, der in der gesamten Inschrift als Imperator Caesar M. Aurellius Antoninus Pius Augustus auftritt, dann der jüngere Sohn, P. Septimius Geta, der mit dem Titel nobilissimus Caesar genannt wird. In der Gestaltung der Schrift ist die Schriftgröße in Z. 4 bereits etwas kleiner als in den ersten drei Zeilen. Deutlich kleiner, fast auf die Hälfte reduziert, ist die Schrift von Z. 5, in welcher C. Fulvius Plautianus als viertes Mitglied der Kaiserfamilie genannt wird. Diese Stelle in der inscriptio ist die einzige Nennung von Plautianus mit seinem vollständigen Namen. ${ }^{2}$ Alle weiteren Erwähnungen beschränken sich auf seinen Titel $P R$ (aefectus) $P R$ (aetorio).

Die Namen von Geta und Plautianus fielen einer damnatio memoriae zum Opfer. Die Spuren der Tilgung sind in Z. 4 f. gut sichtbar; dabei fällt auf, dass die Ausführungen der Rasuren unterschiedlich sind, ein Indiz dafür, dass sie zu unterschiedlichen Zeiten ausgeführt wurden. Der Name und Titel von Geta wurde 2 I I nach der Ermordung Getas getilgt, der Name und Titel von Plautianus schon Anfang des Jahres 205 nach seinem Fall unter Septimius Severus. Der Name von Plautianus wurde nicht mit

I Act. Aug. 53.

2 Siehe zu der neuen Ergänzung des Namens mit nomen gentilicium den Kommentar zur Textkonstitution (Kap. B.3) zu dieser Stelle, S. $342 \mathrm{f}$. 
derselben Konsequenz in der Inschrift getilgt, wogegen die Tilgung des Namens von Geta nur an einer Stelle übersehen wurde. ${ }^{3}$

Die vier Mitglieder des Kaiserhauses sind als Veranstalter der siebenten ludi saeculares aufgeführt, wobei durch die Gestaltung ihrer Namen eine deutliche Hierarchie erkennbar ist.

5-25 Diese Zeilen geben ein für die ludi saeculares wichtiges Dokument wieder. Das Datum ist nicht rekonstruierbar. In den Zwischenraum PRI$D[\cdot 7 \cdot] I A S$ können verschiedene Daten eingesetzt werden. Klar ist aber, dass der hier geschilderte Vortrag des Dokuments im Jahr 203 stattfand, weil der Magister des Kollegiums der Quindecimviri für das Jahr 203, Manilius Fuscus, den Text vorliest. ${ }^{4}$ Es handelt sich um einen Antrag der Priesterschaft der Quindecimviri an den Senat, ludi saeculares auszurichten. Dazu werden die Voraussetzungen für ludi saeculares festgehalten. Der Text nennt den Ablauf des alten saeculum (Z. 7), die Verantwortung das alte saeculum mit der Feier der ludi saeculares abzuschließen (Z. $7 \mathrm{f}$.), das Orakel der Sibylle (Z. 8), das Bemühen, für den korrekten Ablauf der Feier mit den geforderten Opfern zu sorgen (Z. 8f.). Trotz des fragmentarischen Textzustandes ist erkennbar, dass die oben erwähnten vier Mitglieder der Kaiserfamilie direkt angesprochen werden (Z. ıo), die Erfordernisse von Säkularspielen zu gewährleisten. In den folgenden Zeilen werden einige Schlagwörter der ludi saeculares genannt, deren genauer Zusammenhang sich jedoch nicht rekonstruieren lässt. Es geht um Erinnerung (Z. Iо), Fruchtbarkeit (Z. I I), zahlreiches Publikum (Z. I I), Unwetter und Ernteausfälle (Z. I2), Auspizien (Z. I2). In Z. I 3 wird mit der Erwähnung des 956. Jahres nach Gründung der Stadt das Jahr der Spiele genannt. Durch die Nennung dieser Jahreszahl wird klar, dass dieser Text im Jahr vor den Säkularspielen vorgelesen sein musste, möglicherweise zu einem Zeitpunkt, als noch keine eponymen Konsuln gewählt waren. Z. I4 erwähnt die von Augustus etablierte Reihe von Säkularfeiern, deren erste im 297. Jahr nach Gründung der Stadt stattfand. Die severische Säkularfeier schloss sich dieser Reihe als die siebente an. ${ }^{5}$ M. Valerius und Spurius Verginius, die in Z. I 4 genannt werden, waren die Konsuln des Jahres 456 v. Chr., in welchem nach der augusteischen Konstruktion die ersten ludi saeculares stattfanden. ${ }^{6}$

3 PR. PR. ist nicht einer Rasur zum Opfer gefallen in den Zeilen 223, 229, 230, 250,253 , 266, 282; Geta ist nur in Z. 230 nicht unlesbar gemacht.

4 Pighi (I94I) I40 schlägt wegen der erhaltenen Endung des Monatsnamens einen nicht bestimmbaren Tag im März, Mai, Juni oder Juli vor. Birley (I999) I 57 hält März, Juni oder Juli für möglich.

5 Siehe Kap. A.5, S. I67.

6 Die augusteische Reihe forderte ludi saeculares in den Jahren: 456-346-236- I 26 - I7 v. Chr. 
Auch die folgenden Zeilen I 5-25 sind schwer verständlich, es ist die Rede von feriae, vom 660. Jahr seit Begründung der Spiele, also vom Jahr 204 n. Chr., dem Jahr der severischen ludi saeculares, Auspizien (Z. Isf.). Z. I7 nennt Septimius Severus mit Caracalla, Geta und Plautianus als künftige, nach dem Willen der Götter handelnde Veranstalter der siebenten Iudi saeculares. In den Zeilen I8-20 sind weitere Elemente der Säkularspiele erwähnt wie Knaben, die Zeit der Jugend, Iuno Lucina, 7 die als Helferin bei Geburten verehrt wurde, ehrfürchtige Stimmen (von Chören ?), Priester, der Imperator, die Ergebenheit der Menschen, der Ablauf einer bestimmten Anzahl von Jahren und weiteres Gedeihen. Es handelt sich eher um ein priesterliches Schreiben als um einen formal gestalteten Antrag. Darin werden sämtliche Voraussetzungen der ludi saeculares vorgetragen, die das Abhalten der Feier nahelegen.

Der Text schließt mit einer Passage (20-25), die wohl ein Willensbekenntnis des Kaisers ist und ebenfalls von Manilius Fuscus vorgelesen wurde. ${ }^{8}$ Darin wird versichert, dass unter Berufung auf das Orakel der Sibylle, nach Ablauf der geforderten i I J Jahre künftig Säkularspiele unter Leitung von [Severus] und seinem Sohn Aurellius Antoninus, wie Caracalla in der ganzen Inschrift genannt wird, veranstaltet werden sollen. Wegen des fragmentarischen Textzustandes wird aus Z. 2 I nicht klar, ob auch die anderen Mitglieder der Kaiserfamilie ebenfalls in diese Botschaft einbezogen sind. Da jedoch in den Zeilen 58-6I und 6I-63 zwei Briefe von Septimius Severus und seinem ältesten Sohn zitiert werden, ist anzunehmen, dass bei den vorbereitenden Beschlüssen der Säkularspiele Geta und Plautianus keine Rolle spielten.

Die künftigen Spiele sollen unterbrochen sein von Fröhlichkeit und Freude für die Menschen (Z. 2 If.). Gleichzeitig werden die Patres Conscripti, die Senatoren, aufgefordert, im Hinblick auf künftiges Bevölkerungswachstum (Z. 22) dafür zu sorgen, dass auf Kosten der Öffentlichkeit (Z. 23) die Verehrung der Götter und Wohlergehen des Volkes gewährleistet wird. Grundlage künftiger Planung sind die überlieferten Orte der religiösen Verehrung, die zu besuchen sind (Z. 24).

Trotz des fragmentarischen Textzustands kann davon ausgegangen werden, dass nur diese beiden Dokumente aus dem Jahr vor dem Beginn der Säkularspiele als Ausgangspunkt für die Planung und Organisation der Feier zitiert werden.

7 Im carmen saeculare hat Horaz den weitgehend unbekannten Namen von Ilithyia mit Lucina umschrieben. Siehe Kap. A.I I, S. 248. Vielleicht liegt auch hier ein Hinweis auf die Aufführung des carmen saeculare vor.

8 Act. Sev. 20-25. Dem Kollegium der Quindecimviri lag wahrscheinlich ein Dokument von Septimius Severus vor, in welchem er der Abhaltung von Säkularspielen zustimmte. 
26-46 Ein sehr langes vacat, das sich bis an das Ende der Zeile erstreckt, grenzt diesen Text vom vorangehenden ab. Es handelt sich um die Verlesung eines Textes aus einem manuarium, einem Handbuch, wohl über die Organisation von Säkularspielen. Calpurnius Maximus, ${ }^{9}$ ein Senator und Quindecimvir, dessen Ehefrau eine der Io9 Matronen der Säkularspiele war, ${ }^{10}$ hat den Text vorgelesen. Zeit und Ort des Anlasses sind nicht angegeben; Pighi geht zu Recht davon aus, dass die Lesung mit anschließendem Antrag ebenfalls vor dem Senat an demselben Tag stattfand wie die Lesung von Manilius Fuscus (Z. 5-20), weil für eine neue Angabe kein Raum vorhanden ist. Die Gestaltung der Inschrift räumt dem von Calpurnius vorgelesenen Text großes Gewicht ein, denn er beginnt (Z. 26) und schließt (Z.46) mit einem sehr langen vacat.

Eingeleitet wird der Text mit der Verkündung, dass die sanctissimi piissimique principes dem Antrag der Quindecimviri zugestimmt haben, Säkularspiele auszurichten an den dafür bestimmten Tagen im folgenden Jahr (Z. 26-28). Anschließend werden einige Vorgaben für Säkularspiele vorgetragen, die Septimius Severus und Caracalla mit den Quindecimviri beschlossen haben: die Übernahme der Ausgaben durch die Staatskasse (Z. 29), das Aussetzen der Gerichtsbarkeit (Z. 29), die Aufhebung der Trauer für Matronen (Z. 3o), die Errichtung einer Marmorsäule mit Inschrift und wahrscheinlich auch einer bronzenen Inschrift (Z. $3 \circ$ f.). All diese Voraussetzungen für die ludi saeculares sind aus der augusteischen Inschrift bekannt. ${ }^{\text {I }}$

$\mathrm{Da}$ in den folgenden Zeilen der Text stark beschädigt ist, sind keine konkreten Bestimmungen erkennbar. Wahrscheinlich war der von Calpurnius vorgelesene Text in Z. 33 abgeschlossen, worauf ein längeres vacat hinweist. In welchem Zusammenhang der nun folgende Text mit mehrmaliger Nennung der Mitglieder der kaiserlichen Familie (Z. 34, 4I f.) zu verstehen ist, bleibt unklar. In den Zeilen $42 \mathrm{f}$. wird noch einmal auf einige der üblichen Bestimmungen von Säkularspielen eingegangen, wie dem r rojährigen Abstand zwischen zwei Feiern und alle Priesterkollegien und Senatoren (Z. 43 f.). Abgeschlossen ist diese Passage mit Sicherheit in Z. 46, wofür ein sehr langes vacat und der vorgezogene linke Rand der folgenden Zeile sprechen.

$47 \mathrm{f}$. Mit dem Wort [ce]NSEO wird ein Antrag an den Senat eingeleitet, allerdings ist nicht klar, wer diesen Antrag formuliert. Z. 47 erwähnt das Wort COMMENTARIVM, womit die bereits in Z. zof. erwähnte Errichtung

$9 \mathrm{PIR}^{2} \mathrm{C} 277$.

Io Act. Sev. I 88 f. Siehe Kap. B.5, S. 404; Matrone Nr. 6.

I I Übernahme der Kosten aus der Staatskasse Act. Aug. 63; Aussetzen der Gerichtsbarkeit Act. Aug. 39 (FERIAE); Aufhebung der Trauer der Matronen Act. Aug. I IO-I I 4; Errichtung der Inschriften Act. Aug. 60, 6I. 
einer marmornen und bronzenen Inschrift mit einem Bericht über den Ablauf der Spiele gemeint ist. In Z. 48 geht es um PECVNIAM, möglicherweise wieder um die Finanzierung der Inschriften oder der geplanten Spiele. Ein längeres vacat, welches sich bis zum Ende der Zeile erstreckt, beschließt in Z. 48 diese Passage.

49-53 Hier beginnt wieder mit dem vorgezogenen linken Rand ein neuer Abschnitt. Es handelt sich um Maßnahmen oder Beschlüsse, die in einer Versammlung am I 3. November des Jahres 203 erfolgten, etwa ein halbes Jahr vor Beginn der ludi saeculares. Die Sitzung fand auf dem Palatin statt, anwesend waren einige Mitglieder des Kollegiums der Quindecimviri, von denen die Namen von Crescens Calpurnianus (Z. 5o) und Atulenus Rufinus (5I) erhalten sind. Der zur Verfügung stehende Raum in diesen Zeilen reicht nicht für eine Aufzählung aller Mitglieder des Kollegiums aus. Es lassen sich keine Aussagen über den Inhalt machen. Ein langes vacat in Z. 53 macht den Abschluss dieses Abschnitts deutlich.

54-58 Z. 54 als größtenteils unbeschriebene Zeile ist für die Textgestaltung der severischen Inschrift ungewöhnlich. ${ }^{12}$ Vielleicht wurde damit auf das nun folgende Edikt aufmerksam gemacht. Dieses wird mit der Titulatur von Septimius Severus, Caracalla und Geta in den Zeilen 55-57 eingeleitet. Alle drei sind mit voller Titulatur und Filiation im Nominativ genannt, Plautianus ist nicht erwähnt, wie in allen Edikten dieser Inschrift. ${ }^{\mathrm{I}}$ Über den Inhalt können keine Aussagen gemacht werden. Ich habe mich entschieden, die von Pighi eingefügte Z. $57^{\text {a }}$ mit dem Zusatz der Quindecimviri als Urheber des Edikts zu streichen, eine Ergänzung, die auch in Z. 58 möglich wäre. ${ }^{14}$ Es liegt nahe zu vermuten, dass während oder anschließend an die Sitzung des Kollegiums vom I3. November die beschlossenen Maßnahmen für die ludi saeculares per Edikt angeordnet wurden. Da die Inhalte der Sitzung nur etwa zwei Zeilen (Z. 52f.) beanspruchen, halte ich es für möglich, dass auch das Edikt sehr kurz war.

58-6I Das nächste zitierte Dokument des Protokolls der Planung der Säkularspiele stammt vom I 5. April 204. Es zitiert einen Brief von Septimius Severus und Caracalla an das Kollegium der Quindecimviri. Diese werden gebeten, am 25. Mai, also sechs Tage vor Beginn der ludi saeculares, auf dem Palatin im Tempel des Apollo zu erscheinen, um die fünf DreierGruppen auszulosen, die auf fünf verschiedenen Tribünen in der Stadt reinigende Rauchwaren an die Bevölkerung verteilen sollen. ${ }^{\text {Is }}$ Vorgelesen

I 2 Vgl. Act. Aug. I Iо; die Einleitung des Edikts wurde in etwas größeren Buchstaben umgeben von längeren vacat in die Mitte der Zeile gesetzt. Diese Art der Gestaltung wäre auch in Act. Sev. 54 möglich.

I3 Act. Sev. 7I-79; I I-I I7; 209-222.

I4 Pighi hat die Edikteinleitung von Z. 209 als Vorbild genommen.

is Siehe den Sachkommentar zu Act. Aug. 7-I I. 
wurde dieser Brief von Iulius Pompeius Rusonianus, dem Magister des Kollegiums im Jahr 204 n. Chr.

6I-63 Direkt anschließend wird ein zweiter Brief von Severus und Caracalla an das Kollegium zitiert. Die ersten Bestimmungen in diesem Brief sind nicht mehr erhalten, nur die Angabe, mit welchen Duftstoffen die Matronen das Supplikations-Gebet am 2. Juni vortragen sollen, ist lesbar. Diese Angabe macht deutlich, wie detailliert die Planung der severischen ludi saeculares vorgenommen wurde und wie detailgetreu die Aufzeichnungen sind. Über Duftstoffe bei Gebeten ist außer an dieser Stelle nichts bekannt, es handelt sich wahrscheinlich um das Verbrennen von Weihrauch oder ähnlicher Stoffe.

64-7I Diese Zeilen geben ein Protokoll der oben erwähnten Sitzung des Kollegiums der Quindecimviri wieder, an welchem die Auslosung stattfand. Interessant an diesem Text ist die Präsenzliste des Kollegiums, die die Namen aller Mitglieder umfasste. Von den 20 Mitgliedern des Kollegiums sind in dieser Liste I3 Namen erhalten, die zwischen den Fragmenten liegenden Lücken lassen auf sieben fehlende Namen schließen. Exakt sieben Namen von Quindecimviri lassen sich an anderen Stellen der Inschrift identifizieren, womit sich eine vollständige Liste der Quindecimviri herstellen läßt. ${ }^{16}$

Dazu kommen die vier Mitglieder der Kaiserfamilie, die ebenfalls Quindecimviri waren. Die augusteische Inschrift enthält eine Gesamtliste des Kollegiums am Ende der Inschrift, die eingeleitet ist mit den Worten: HAEC OMNIA PERACTA SVNT XV[vir. s. f. Die severische Inschrift hat aus Platzmangel einen Teil des Textes auf die rechte Seite des Marmorblockes verlegen müssen. Es ist trotz des fragmentarischen Zustandes dieses Teils erkennbar, dass kein Gesamtverzeichnis der Quindecimviri am Ende der Inschrift vorlag. Die einzig mögliche Stelle für eine Gesamtliste des Kollegiums liegt in den Zeilen 65-68 vor. Die Hierarchie innerhalb des Kollegiums war wie üblich nach dem Eintrittsdatum eines Mitglieds gegeben. ${ }^{17}$

Die Auslosung der Dreier-Gruppen für die Verteilung der suffimenta und die Entgegennahme der Früchte lässt sich gemäß Pighi aus dem Text rekonstruieren. ${ }^{18}$ An folgenden fünf Orten wurden Tribünen errichtet:

I. in Palatio in tribunali Angustorum nostrorum, quod est in area aedis Apollinis (Z.68)

2. in tribunali, quod est ad Romam Quadratam (Z.69)

I6 Siehe den Kommentar zur Textkonstitution (Kap. B.3) zu dieser Stelle und Kap. B.5, S. $404 \mathrm{ff}$.

I7 Siehe Kap. B.5, S. 404 .

I 8 Pighi S. $237 \mathrm{ff}$. 
3. in Capitolio ante aedem Iovis O. M. (Z. $70 /$ nach Act. Aug. 30)

4. ante aedem Iovis Tonantis (Z. $70 /$ nach Act. Aug. 3 I)

5. (in Aventino) ante aedem Dianae (nach Act. Aug. 32). ${ }^{19}$

Die Verteilstationen waren nicht weit voneinander entfernt, und auf jedem Podest saß eine Dreiergruppe von Quindecimviri:

I. in Palatio: Ulpius Soter cos. des., Pollienus Auspex, Salvius Tuscus

2. ad Romam Quadratam: Nonius Mucianus, Aiacius Modestus, Atulenus Rufinus

3. ante aedem Iovis O. M.: [...], [...], Ofidius Macedo

4. ante aedem Iovis Tonantis: [...], Fulvius Fuscus Granianus, Vetina Mamertinus

5. ante aedem Dianae: [...], [...], Cocceius Vibianus.

Kein Mitglied der Kaiserfamilie ist als Besetzung eines dieser Podeste vorgesehen. Aus den Zeilen 79-90 kann man schließen, dass sie kein Podest ständig besetzten, sondern an allen fünf Podesten eine Zeitlang suffimenta verteilten und dann zum nächsten Podest weiterzogen.

Geta und Plautianus sind nicht als Anwesende an der Sitzung zur Auslosung erwähnt. Aus der Anwesenheitsliste von Z.65 geht klar hervor, dass nur die beiden Augusti, Severus und Caracalla, präsent waren.

71-79 Dieser Abschnitt enthält ein Edikt über den Ablauf der Spiele. Das Edikt ist ohne Datum eingeleitet, die Angabe SORTIBLus ductis weist darauf hin, dass es ebenfalls am 25. Mai, dem letzten genannten Datum erlassen wurde, also direkt nach der Verlosung. Erlassen wurde das Edikt, wie bereits das Edikt von Zeile 54-58 von Severus, Caracalla, Geta und den Quindecimviri. Dieses Edikt verkündet dem Volk, an drei Tagen und drei Nächten zu den Säkularspielen zusammenzuströmen (Z. 75). Es gibt bekannt, dass dieser Anlass allgemeiner Fröhlichkeit allen Bürgern Gewinn bringen wird (Z. 76), dass während des Festes die Gerichtsbarkeit ruhen soll, aber nach 30 Tagen erneut einsetzt (Z. 77). Interessant ist die Mahnung des Edikts, dass Bürger, Hausbesitzer und Ladenbesitzer in den Nächten der Spiele Maßnahmen treffen sollen, dabei können sie auf Unterstützung von Soldaten zählen (Z. $78 \mathrm{f}$.). Welcher Art diese Maßnahmen waren, ist wegen der relativ großen Lücken nicht mehr erkennbar, wahrscheinlich waren Sicherheitsvorkehrungen gemeint. Daneben müssen noch weitere Bestimmungen in diesem Edikt enthalten sein. Es fehlt ein Hinweis an die Bevölkerung, dass sie bereits vor Beginn der ludi saeculares an fünf Orten in der Stadt Räucherwerk zur rituellen Reinigung ihrer Wohnungen holen sollen. Auch die anschließende acceptio frugum,

I9 Vgl. den Sachkommentar zu Act. Aug. 7-I I, S. 86. 
an welcher Bürger Erstlingsfrüchte an denselben Tribünen abliefern können, ist nicht in diesem Text erkennbar. ${ }^{20}$ Es ist möglich, dass Bestimmungen, die die Ausübung der Riten der ludi saeculares betrafen, durch einen Herold ausgerufen wurden, wie er in der augusteischen Inschrift und durch Münzen belegt ist. ${ }^{21}$

79-90 Hier werden die ersten Handlungen, die zu den ludi saeculares gehörten, protokollartig geschildert. Das angegebene Datum ist der 26. Mai. Wie bei den augusteischen Spielen sind die Daten für die Verteilung der suffimenta für den 26., 27. und 28. Mai vorgesehen. Die acceptio frugum fand im Jahr I7 v. Chr. am 30. Mai statt, was auch für die severische Feier angenommen werden kann. ${ }^{22}$

Die unter Beteiligung großer Menschenmengen stattfindende Verteilung der suffimenta wurde von jeweils drei Quindecimviri an den oben erwähnten fünf Orten durchgeführt. Als erste sind die Imperatoren Septimius Severus und Caracalla als Verteiler auf der Tribüne des Palatins erwähnt (Z. 79f.). Aus dem zerstörten Text in Z. 79 geht nicht mit Sicherheit hervor, ob sie zusammen mit Geta eine kaiserliche Dreiergruppe bildeten; ${ }^{23}$ da sie aber jeweils von Dreiergruppen der Quindecimviri abgelöst wurden, ist es wahrscheinlich. Die Verteilung war nach den erhaltenen Aufzeichnungen zu schließen nach sozialem Stand organisiert: zuerst für Senatoren und Priester, anschließend für den Ritterstand und zuletzt für den populus Romanus. ${ }^{24}$ Septimius Severus und seine Söhne blieben nicht während der vorgesehenen drei Stunden, ${ }^{25}$ sondern wurden von den für diesen Ort ausgelosten Quindecimviri nach einiger Zeit abgelöst, um zur nächsten Verteilstelle weiter zu ziehen und alle fünf Stationen besuchen zu können. In Z. $8 \mathrm{I}$ ist das ablösende Trio auf dem Palatin namentlich erwähnt (Z. 8I). In Z. 82 wird nur gesagt, dass die übrigen Quindecimviri an den jeweils ausgelosten Stellen in der toga praetexta im Einsatz waren, ihre namentliche Nennung folgt erst in den Zeilen 85-90. Vorher, in den Zeilen 8 I-85, ist die Prozession der Severer von Tribüne zu Tribüne ausführlich beschrieben. Trotz der Lücken im Text können wir viermal suffimenta diviserunt lesen (Z. 80, 82-84). Sicher war auch die Verteilung auf der fünften Tribüne verzeichnet. In Z. 84 ist die Verteilung der suffimenta durch die Kaiserfamilie abgeschlossen. Mit

20 Siehe den Sachkommentar zu Act. Aug. 7-I I, S. $84 \mathrm{ff}$.

2 I Siehe den Sachkommentar zu Act. Aug. C I 5-17, S. $78 \mathrm{ff}$.

22 Act. Aug. 67 und 82.

23 Allerdings ist Geta bei der Auslosung der Besetzung der Tribünen nicht unter den Anwesenden erwähnt, siehe Act. Sev. 65 .

24 Act. Sev. 79-8 I. In der Lücke von Z. 80 vermutet auch Scheid (20I3) I 3 I die Nennung der sozial über dem Ritterstand stehenden Angehörigen des römischen Volkes.

25 Act. Sev. 89. 
welcher Handlung Septimius Severus in Z.85 verbunden werden muss, wird angesichts des schlechten Textzustands nicht klar. In Z. 86 schließen die Namen der 12 noch nicht genannten Quindecimviri die Verteilung der suffimenta ab. In Z. 89 werden mit den römischen Zahlen die Stunden angegeben, an welchen die Verteil-Aktion stattfindet, von der neunten bis zur zwölften Stunde, das heißt in den frühen Nachmittagsstunden der drei dafür vorgesehenen Tage. Wir haben allerdings keinen inschriftlichen Hinweis, dass die Verteilung bei den severischen ludi saeculares auch an drei Tagen durchgeführt wurde.

Bei der Aufstellung der Fragmente liegt zwischen den Zeilen 86 und 87 ein größerer Zwischenraum, der etwa so Zeilen aufnehmen könnte. Da in Z. 87 die Aufzählung der verbleibenden vier Gruppen von Quindecimviri fortgesetzt wird, liegt höchstens eine Zeile dazwischen; möglicherweise fand die fünfte Gruppe in diesen Zeilen ihre Erwähnung. ${ }^{26}$

90-96 Da der linke Rand in diesem Teil der Inschrift erhalten ist, kann man davon ausgehen, dass bis Z. 97 keine neue Handlung mit einem neuen Datum geschildert wird. Deshalb müssen die Handlungen, die in den Zeilen 90-96 geschildert werden, wohl in Zusammenhang mit der Verteilung der suffimenta gesehen werden. Dafür spricht die Erwähnung der scipiones ebornei in Z. 91. Diese elfenbeinernen Stäbe waren rituelle Gegenstände und wurden während verschiedener ritueller Handlungen getragen. ${ }^{27}$ Eine domitianische Münzabbildung läßt schwach erkennen, dass bei der Verteilung der suffimenta der Kaiser auf dem Podest mit der rechten Hand dem vor ihm stehenden Bürger etwas übergibt und in der linken gesenkten Hand einen Gegenstand hält, der ein solcher Elfenbeinstab sein könnte. ${ }^{28}$

Die Verteilung der Rauchwaren wurde von Severus gemäß den Zeilen $92 \mathrm{f}$. mit einem Gebet begleitet oder abgeschlossen, dessen Inhalt sich nicht rekonstruieren läßt. Angesprochen waren von den an den Säkularspielen verehrten Göttern die Götter der Opfer am Tag, also Iuppiter, Iuno und Apollo, vielleicht auch Diana. Der Wortlaut des Gebets entspricht nicht dem der Opfergebete. Auffällig ist aber, dass neben der res publica die Kaiserin Iulia Domna in den Gebetstext einbezogen wurde (Z. 95 f.).

Der Abschnitt über die Verteilung der suffimenta schließt wieder mit einem beschreibenden Teil, worauf das Verb procu]RAVERVNT hinweist.

$26 \mathrm{Zu}$ den verschiedenen Gruppen von Quindecimviri siehe den Sachkommentar zu Z. 68-7i, S. 37if.

27 Act. Sev. 254 überliefert, dass Severus und Caracalla bei der abschließenden pompa solche Elfenbeinstäbe trugen.

28 Auf einer Münze zur acceptio frugum ist auch ein Gegenstand in der Hand des agierenden Kaisers zu erkennen. Siehe Anhang, Abb. $9 b$. 
97-99 Zwei Tage nach der Verteilung der suffimenta fand an den augusteischen Indi saeculares die Annahme der Erstlingsfrüchte statt. ${ }^{29}$ Dabei brachten die Bürger an dieselben Tribünen, an denen sie die suffimenta erhalten hatten, Erstlingsfrüchte und leerten diese in Körbe. Der vorgezogene linke Rand der severischen Inschrift macht den Beginn einer neuen Handlung kenntlich. Das Datum ist nicht genannt und mit INDE sehr vage. Möglicherweise bezieht sich das vorangegangene Gebet auch auf die acceptio frugum, sodass die Gruppe aus Kaiserfamilie und Quindecimviri sich nach dem Gebet zu den Tribünen bewegte. Die acceptio frugum erstreckt sich in der severischen Inschrift nur über drei Zeilen (Z. 97-99) und ist sehr verstümmelt. Der Abschnitt wurde sicher unter Hinweis auf die ausführliche vorangegangene Darstellung der Verteilung der suffimenta abgekürzt, um nicht noch einmal die Wanderung des Kaisers und seiner Söhne von Tribüne zu Tribüne zu schildern. Als Empfänger traten neben dem Kaiser und seinen Söhnen wieder die fünf Dreiergruppen von Quindecimviri auf. Die abgegebenen Früchte wurden in Körbe zu Füßen des Kaisers und seiner Söhne oder der Quindecimviri gelegt (Z. 99), wahrscheinlich - wie in der augusteischen Inschrift festgehalten ist - nach Arten voneinander getrennt. ${ }^{\circ}$ In der severischen Inschrift sind diese Früchte noch einmal erwähnt (Z. 258f.). Sie wurden Wagenlenkern, Kunstreitern und dem Aufsichtspersonal nach dem feierlichen Abschluss der ludi saeculares am 3. Juni verteilt.

ıoof. Auch diese Zeilen sind sehr fragmentarisch und enthalten die Darstellung mehrerer ritueller Handlungen. Ein Datum ist nicht angegeben; die beschriebenen Handlungen fanden nach Abschluss der acceptio frugum statt. ${ }^{31}$ Am Anfang (Z. Ioof.) handelt es sich um den Abschluss der Sammlung der abgegebenen Früchte, die wohl ins Tarentum gebracht und dort deponiert wurden, weil sie nach Abschluss der ludi saeculares dort verteilt wurden..$^{32} \mathrm{Im}$ Tarentum spielen sich auch die im Anschluss geschilderten Handlungen ab (Z. ıо f.). Möglicherweise fand der Transport der abgegebenen Früchte wieder in einem feierlichen Zug ins Tarentum statt. Dort führt der Magister des Kollegiums Pompeius Rusonianus eine lustratio durch, um das Heiligtum rituell für die folgenden Säkularopfer vorzubereiten. Darunter sind nicht nur Handlungen im Sinne einer rituellen Reinigung zu verstehen. ${ }^{33}$ Das lustrum stellte ein Mittel dar, um ein Gelände nach vorbereitenden reinigenden und nötigenfalls entsühnenden Handlungen mit Umgangsriten für die folgenden Opfer und

29 Siehe den Sachkommentar zu Act. Aug. 7-I I.

30 Act. Aug. 69: GENERATIM CONFERRENT.

3 I Bei den augusteischen Säkularspielen fand die acceptio frugum am 30. Mai statt.

32 Act. Sev. $258 \mathrm{f}$.

33 Scheid (2016a) 203-209. 
Feiern zu bestimmen und den Ort nach Jahren der Verlassenheit wieder seiner religiösen Bestimmung zuzuführen. Ein Aufräumen und eine Reinigung des ungenutzten Geländes im Heiligtum von Dis Pater und Proserpina im Tarentum wird schon vorher durch Sklaven stattgefunden haben.

In Z. I02 beginnt ein Text, den der Magister Pompeius Rusonianus zitiert. Es scheint sich um ein Gebet oder eine Fürbitte zu handeln, in welcher Septimius Severus, Caracalla und Geta als künftige Veranstalter der Säkularspiele einbezogen sind (Z. I03-I06). Die Namen der angesprochenen Götter sind nicht erhalten, die Endungen der Verben in Z. 106 (LVSTRE[ti]S, PIE[t]IS, PVRGETIS) weisen auf mehrere Gottheiten hin. Dieser Text endet möglicherweise mit einer erneuten Nennung des Kaisers und des Staates (Z. i Iо: $A V G$. und i i : IMPERIO).

I I I-I I 8 Die Zeilen I I I-I I 4 enthalten ein Datum, die Titulatur und Filiation des Kaisers und seiner Söhne und stellen die Einleitung zu einem Edikt des Kaisers, seiner Söhne und der Quindecimviri dar. Der mit M]ARTIAS lesbare Rest des Datums passt nicht in die chronologische Reihenfolge der Schilderung der Ereignisse. Offenbar wird hier ein Edikt zitiert, das schon Ende Februar oder Anfang März erlassen worden war. Entweder ist es bei der chronologischen Schilderung vergessen worden oder sein Inhalt betrifft die nun folgenden Handlungen, sodass es zusammen mit diesen zitiert wird. Ein längeres vacat am Ende von Z. I I 4 hebt das Edikt im fortlaufenden Text der Inschrift hervor.

Nach der Edikteinleitung wird der Inhalt des Edikts mit vorgezogenem linkem Rand kenntlich gemacht. Es wird nicht klar, wann genau der Text des Edikts abgeschlossen ist und das nächste Ereignis, das sacrum bostiae praecidaneae, anfängt. Auch der Inhalt dieses Edikts ist nicht mehr erkennbar. Aus Worten wie ORDINEM, PROVIDENTIA, c]OM$M V[n \cdot I \circ \cdot]$, POPVLI ROM[ani, PRAECE[dere vermute ich, dass es um Maßnahmen zum geordneten Ablauf der Feierlichkeiten mit großen Menschenmassen geht. Vielleicht haben die Quindecimviri von ihren Tribünen aus noch etwas verteilt (Z. I I f.). Das Ende des Edikts vermute ich wie Pighi in Z. I 8 .

I I-I 37 Diese lange Textpassage ohne Datumsangabe schildert das sacrum bostiae praecidaneae, mit welchem die ludi saeculares wohl am Tag oder nur Stunden vor dem nächtlichen Opfer für die Moiren begannen. Der Ort dieses Voropfers ist zwar nicht lesbar, die neuen Ergänzungen von Coarelli und mir stützen aber die Annahme, ${ }^{34}$ dass dieses Opfer im Tarentum stattfand. Eine Prozession führte vom Palatin durch die via sacra

34 Coarelli (1997) I 30. Siehe den Kommentar zur Textkonstitution (Kap. B.3) zu dieser Stelle und Kap. B.6, S. 463 f. 
(Z. I 8 f.), wahrscheinlich wie die abschließende Prozession auch durch den neu errichteten Triumphbogen des Septimius Severus, vorbei am theatrum Marcelli und der porticus Octaviae (Z. I 19), deren aufwendige Renovation gerade abgeschlossen war. Weiter bewegte sich der Zug vorbei am Odeum (Z. I20) in Richtung Tarentum, wo das Opfer am mit Lorbeergirlanden geschmückten Ufer des Tiber (Z. I 20) abgehalten wurde. ${ }^{35}$ Während des Opfers waren die Oberpriesterinnen der Vesta, $\mathrm{Nu}$ misia Maximilla und Terentia Flavola, anwesend, die sicher auch an der Prozession teilgenommen hatten. Diese beiden Priesterinnen sind auch als Anwesende während des Supplikationsgebets der Matronen erwähnt (Z. I84f.). Der Inhalt der beiden Zeilen I22f. bleibt im Dunkel, möglicherweise waren dort weitere Beteiligte der Prozession und Opferzeremonie erwähnt..$^{36}$

Z. I 24 markiert mit vorgezogenem linkem Rand einen neuen Abschnitt. Die bisherigen Editoren vermuteten hier den Beginn des Gebetstextes mit der Anrufung von Iuppiter, weswegen die folgenden Epitheta im Vokativ ergänzt wurden. Ich bin mir nicht sicher, ob in dieser Nennung von Iuppiter schon die Anrufung des Gottes zu sehen ist, da es in den folgenden Zeilen eindeutig noch nicht um einen Gebetstext geht. In Z. I 25 f. ist noch einmal von Septimius Severus als Augustus und Pontifex Maximus die Rede, am Ende von Z. I26 könnten die Buchstaben JMO$T O R-\mid V M$ vielleicht mit dem Verb submovere darauf hinweisen, dass der Weg für Priester und Magistrate freigemacht wurde. In jedem Fall ist es nicht möglich, dass in den Zeilen I $25 \mathrm{f}$. der Text eines Opfergebets zitiert wird, möglicherweise war das Gebet wie das Moirengebet durch Angaben zur Durchführung der Riten unterbrochen. ${ }^{37}$

Am Ende von Z. r 27 f. sind eindeutig Reste von Wörtern aus einem Gebetstext sichtbar, es handelt sich um die Formel uti] VOBIS $\mid$ IN IJllis libris scriptum est. Davor, noch in Z. I27, muss die Anrufung der Götter gestanden haben. Mit der neuen Lesart des Wortes VALE- $\mid$ TVD[inem in Z. I29f. lassen sich große Teile des Gebetstextes rekonstruieren..$^{8}$ Allerdings muss unklar bleiben, welche Götter (außer Iuppiter) angerufen wurden und mit welchen Opfertieren sie geehrt wurden. ${ }^{39}$ Das Gebet des

35 Nach Abschluss des letzten Opfers der ludi saeculares am 3. Juni für Apollo und Diana verzeichnet die Inschrift eine Prozession mit pompa auf derselben Strecke, Act. Sev. 249-254. Siehe Kap. B.6, S. 459ff.; eine severische Münzprägung bildet die geschmückte Opferstätte am Tiber ab, siehe Anhang, Abb. I8.

36 Mögliche Ergänzung für Act. Sev. I 22 f.: praeeunte] $\mid$ A[ntonino.

37 Vgl. den Sachkommentar zu Act. Sev. I38-140.

38 Vgl. den Kommentar zur Textkonstitution (Kap. B.3) zu Z. I 27-1 36, S. 350 f.

39 Für die Nennung aller an den Säkularspielen geehrten Götter wäre durchaus genügend Raum in Z. I 27 vorhanden. 
sacrum hostiae praecidaneae enthielt neben den bekannten Bitten des Opfergebets in Z. I 29-I 3 I andere, nicht mehr rekonstruierbare Elemente. In meinen Ergänzungen berücksichtige ich nur die Bitten, die sich aufgrund der erhaltenen Buchstaben eindeutig einfügen lassen. Das Gebet richtet sich an männliche und weibliche Gottheiten (Z. I3I), wobei zweimal eine Gruppe von weiblichen Gottheiten angesprochen ist (Z. I3 If. und I34f.); möglicherweise sind die bei den Säkularspielen verehrten Moiren und Ilithyien gemeint. Dies legt die Vermutung nahe, dass das sacrum hostiae praecidaneae alle an den ludi saeculares geehrten Gottheiten betraf. Wie in dem Opfergebet an Iuppiter erkennbar ist, ${ }^{40}$ wird als letzte der Bitten des Opfergebets die Bitte um Annahme aller Opfer ausgesprochen, die bei der anschließenden Opferzeremonie jedes einzelnen Tieres wiederholt wird. Diese Wiederholungen werden in der Inschrift nur ein einziges $\mathrm{Mal}$ zitiert, für alle weiteren Opfer abgekürzt. Im fragmentarisch erhaltenen Text ist dies erkennbar durch die zweifache Nennung des Wortes accep] |TRIC[ces. In Z. I 36 ist das Opfergebet abgeschlossen, möglicherweise mit einem vacat. Die anschließende Z. I37, von der nur die Buchstaben IN OMNI[ erhalten sind, hat einen etwas vorgerückten linken Rand, womit der Abschluss der Zitation des Gebets markiert sein kann. Der Text hat nun wieder beschreibenden Charakter, vielleicht wurde in dieser Zeile darauf hingewiesen, dass für jedes Opfertier die vorher beschriebenen Opferriten vollzogen wurden. In der augusteischen Inschrift findet sich kein Hinweis auf ein sacrum hostiae praecidaneae. Dies bedeutet nicht, dass es nicht stattgefunden hat, aber es weist darauf hin, dass - falls ein solches Opfer existierte - es als nicht $\mathrm{zu}$ den ludi saeculares gehörig gewertet und somit nicht in die Inschrift aufgenommen wurde..$^{4}$ Mit der sehr ausführlichen Darstellung des sacrum hostiae praecidaneae und dem vollständigen Zitat des dazugehörigen Opfergebets liegt nicht nur eine Abweichung der severischen Aufzeichnungspraxis vor, sondern wahrscheinlich auch eine Abweichung im Ablauf der Feier. Ebenso scheint die vorher (Z. I I 8-I 20) geschilderte Prozession ein von Septimius Severus neu gestaltetes Element der Säkularfeier zu sein. ${ }^{42}$

I38-I54 In Z. I38, mit weit vorgezogenem linkem Rand, ist das Wort MOERAE gut lesbar. Hier beginnt mit der Anrufung der Moiren das Moirengebet

40 Act. Sev. I 57 für beide Ochsen, Act. Sev. I60 für den ersten, Act. Sev. I63 für den zweiten Ochsen.

4 I Act. Aug. C 9-I4 gehört zu einem rituellen Text im Rahmen der religiösen Vorbereitungen der Spiele. $\mathrm{Ob}$ es sich um ein sacrum hostiae praecidaneae handelt, kann nicht gesagt werden. Lipka (2009) I 50, Anm. 7 geht davon aus, dass ein sacrum hostiae praecidaneae nicht zum Konzept der augusteischen Säkularfeier gehörte.

42 Vgl. Kap. B.6, S. $464 \mathrm{f}$. 
und der eigentliche Beginn der Säkularopfer. Dieses Gebet schließt direkt an das sacrum hostiae praecidaneae an, ohne wie sonst in der Inschrift üblich, auf die Anwesenden und eine Prozession oder einen Ortswechsel zur Opferstätte hinzuweisen. ${ }^{43}$ Das Opfergebet für die Moiren ist nicht in seiner vollen Länge wiedergegeben, sondern die Bitten sind nach der zweiten Bitte mit cetera ut supra abgekürzt. Als erstes Gebet wurde offenbar das Opfergebet für das sacrum hostiae praecidaenae betrachtet, weil es als einziges Opfergebet vollständig wiedergegeben wurde. Dies spricht dafür, dass beide Opfer direkt aufeinander folgten, dass alle Ausführenden und Teilnehmenden sich schon im Tarentum befanden, dass schon einmal ein Opfergebet gesprochen war, und dass die Prozession vor dem sacrum hostiae praecidaneae als feierliche Anfangsprozession der ludi saeculares gedacht war. Demnach könnte das sacrum hostiae praecidaneae am Abend des $3 \mathrm{I}$. Mai stattgefunden haben und direkt daran anschließend im späteren Teil der Nacht vom 3r. Mai auf den I. Juni das Moiren-Opfer. Wie die Münzabbildung zum sacrum hostiae praecidaneae ${ }^{44}$ und Z. I 20 zeigen, hat dieses nicht im Gelände des Heiligtums von Dis Pater und Proserpina im Tarentum stattgefunden, sondern irgendwo in der Nähe am Tiberufer. Das Ufer war für diesen Akt mit Lorbeerkränzen geschmückt worden (rip]AM TIBERIS LAVRVM Z. I20), was auf der Münzabbildung gut erkennbar ist.

Das Moirengebet ist bis zur Abkürzung in Z. 139 nach dem gewohnten Muster formuliert. Mit dem Wort ADDITIS in Z. I 40 verlässt der inschriftliche Text das Zitat des Gebetstextes und gibt eine zusätzliche Angabe zum Opfer. Ob weitere Opfertiere oder Zusätze zum Opfer hinzugefügt wurden, bleibt unbekannt. Die Darstellung des rituellen Aktes schließt mit den Bitten um gnädige Annahme der Opfer von neun schwarzen weiblichen Schafen und neun schwarzen weiblichen Ziegen (I4If.).45 Nicht erhalten ist die Angabe des Opfermodus, der aus der augusteischen Inschrift mit $A C H I V O$ RITV bekannt ist. ${ }^{46}$ In den Zeilen I42-I 44 folgt eine Aufzählung der beim Opfer anwesenden Quindecimviri. Die Aufzählung des gesamten Kollegiums bei der Sitzung zur Auslosung der Gruppen an den Tribünen (Z.65-68) erstreckt sich über circa zweieinhalb Zeilen, eben so viel Raum ist hier vorhanden, es ist also möglich, dass das Priesterkollegium vollzählig an dem ersten Anlass der ludi saeculares präsent war. Wahrscheinlich waren alle Akteure bereits bei der Prozession und dem sacrum hostiae praecidaneae dabei.

43 Siehe. Act. Sev. 222 (Terra Mater), 228 (Apollo, Diana); [I79] (Iuno).

44 Siehe Anhang, Abb. I8.

45 Zum Moiren-Opfer siehe den Sachkommentar zu Act. Aug. 90-100.

46 Act. Aug. 9I. 
Anschließend an das nächtliche Opfer fanden die Spiele in dem provisorisch errichteten Theater neben dem Heiligtum von Dis Pater und Proserpina im Tarentum statt. Um diese Aufführungen geht es in den Zeilen I $45-$ I $49 \cdot{ }^{47}$ Die Erwähnung von Iulia Domna in den Zeilen is If. geht wohl auf ihre führende Rolle bei den sellisternia der Matronen zurück, welche nach jedem Opfer auf dem Kapitol durchgeführt wurden. Es ist $\mathrm{zu}$ wenig erhalten, um detailliertere Aussagen machen $\mathrm{zu}$ können. ${ }^{8}$ Auch Z. I 53 gehört zu den Riten der ersten Nacht, wo weitere Zeremonien mit bereits vorher überlieferten Worten (ISDEM VERBI[s) durchgeführt wurden.

I 54-I7I Ohne erkennbaren Einschnitt in der Darstellung beginnt irgendwo in Z. I 53 oder I 54 die Schilderung der Riten des ersten Tages mit dem Opfer von zwei Ochsen für Iuppiter. Weder Datum noch Ort sind erhalten, es ist aus dem Ablauf der Spiele aber klar, dass sich im Morgengrauen des I. Juni der Zug von Kaiserfamilie, Priestern und zahlreichen Zuschauern vom Tarentum zum Kapitol bewegte. Ein provisorisch errichteter Altar aus Holz ist in Z. I 5 s erwähnt, an welchem diese Opfer vor dem Tempel des Iuppiter Optimus Maximus durchgeführt wurden. Der übliche Kleiderwechsel vor jedem Opfer von toga praetexta zu tunica fimbriata (mit Fransen besetztes Gewand) ist ebenfalls notiert. Diese Kleiderwechsel sind nur in der severischen Inschrift überliefert, aus den erhaltenen Angaben lässt sich schließen, dass alle Opferriten in der tunica fimbriata durchgeführt wurden. Für die Prozessionen und die praefatio vor dem Opfer trugen die Ausführenden eine toga praetexta und einen Kranz. ${ }^{49}$ In Z. I 57 beginnt das erste Gebet, welches von Severus gesprochen wurde (Z. I 5 ) und nach der zweiten Bitte wie üblich mit cetera ut supra abgekürzt ist (Z. I 57 f.). Dieses Opfergebet, an Iuppiter gerichtet, begleitet die immolatio und bittet um die Annahme der beiden Ochsen. Nicht erhalten ist eine Angabe, wer dem Kaiser das Gebet vorgesprochen hat, es wird wie bei fast allen Opferhandlungen sein älterer Sohn Caracalla gewesen sein.

Es folgen nach dem Gebet die Schilderungen verschiedener Riten, die sich um die Vorbereitung der Opfertiere drehten. Der betreffende Text ist stark zerstört. Offenbar wurde mit der rechten Hand etwas an dem ersten Opfertier vorgenommen, vielleicht, wie in Z. I65 für das zweite Opfertier noch lesbar ist, eine Locke von der Stirn des Ochsen geschnitten. Darauf folgt die Bitte um gnädige Annahme des ersten Ochsen (Z. I 59 f.). Weitere

$47 \mathrm{Zu}$ ludi und sellisternia siehe den Sachkommentar zu Act. Aug. I00-Io2.

$48 \mathrm{Zu}$ den Spielen im Tarentum und den sellisternia der Matronen siehe den Sachkommentar zu Act. Aug. 100-IO2.

49 Vgl. Act. Sev. I 56, I79, 223f., 227, 229, 247. 
detaillierte Angaben zum Opferhergang sind verzeichnet, von denen leider nichts mehr erhalten ist. Das Wort IVSSIT in Z. I6I lässt vermuten, dass den Opferdienern entsprechende Befehle erteilt wurden, um die Tötung des ersten Ochsen durchzuführen.

In Z. I62 wird mit IN SECVNDO erkennbar, dass die Schilderung nun das Opfer des zweiten Ochsen betrifft. Die Erwähnung der Quindecimviri in dieser Zeile und die erhaltenen Buchstaben $P V R$ lassen vermuten, dass nach der Tötung des ersten Ochsen der Altar erst gereinigt werden musste. Dank der neuen Lesart mJELIVS] kann bestätigt werden, dass in Z. I63 das Opfergebet für den zweiten Ochsen folgt. Doch in diesem Gebet sind die nach der Begründungsklausel für das Opfer folgenden Bitten anders angeordnet: Es wird nicht wie beim ersten Ochsen am Anfang für die Herrschaft und Souveränität des römischen Volkes gebetet, sondern für das Wohlwollen und die Güte der Götter gegenüber dem römischen Volk, dem Kollegium der Quindecimviri und dem Kaiserhaus. Nach der zweiten Bitte (uti semper Lati]NVS OBTEMPER[assit), die in beiden Iuppiter-Gebeten die gleiche ist, wird auch dieses Gebet abgekürzt mit cetera ut supra. Dies geht aus dem Ende von Z. I64 hervor, wo kein Gebetstext mehr vorliegt, sondern eine haruspicatio angekündigt wird.

Die Zeilen I64f. beschreiben rituelle Handlungen vor der haruspicatio, dazu gehört in Z. 165 das Abschneiden einer Stirnlocke des Ochsen und Bereitstellen eines Kranzes, der dem Tier um den Hals gelegt wurde. Anders als Scheid denke ich nicht an einen Kranz, den die Opfernden trugen, sondern eher an ein Accessoire im Ritus der haruspicatio..$^{\circ}$ Darauf spricht Caracalla seinem Vater Severus ein Gebet vor, das kein Opfergebet ist, sondern wohl die bevorstehende haruspicatio betrifft oder wie Scheid vermutet, ein Gebet zum Opfer von Kränzen ist. Aus den Fragmenten ist nur ersichtlich, dass mit Kränzen, die einem Gott - wohl Iuppiter - gegeben werden, Wohlwollen und Gnade für das römische Volk, das Kollegium der Quindecimviri und das Kaiserhaus erbeten werden. Das Gebet ist in Z. I66 abgeschlossen und die Schilderung der Riten, die zur haruspicatio gehören, wird fortgesetzt. Dazu gehören das dreimalige Besprengen des Altars mit Wasser oder Blut und weitere reinigende Handlungen der Opferdiener (Z. I67f.). ${ }^{\text {I1 }}$ In Z. I68 ist ein Befehl von Septimius Severus zum Wasserholen, der an die Opferdiener gerichtet ist, wörtlich überliefert. Dieses Wasser war zur Reinigung des Opfern-

50 Scheid (2005) Ios erinnert an die Opferung von Kränzen bei den Arvalbrüdern während des Opfers an Dea Dia.

5 I Aus der Inschrift geht nicht hervor, womit der Altar besprengt wurde. Scheid (2005) ıos f. hält ein mögliches Besprengen mit Blut hier für einen in den Quellen einmaligen Fall, der eine Parallele zu griechischen Opferriten belegen würde. 
den und zum Besprengen der Opfertiere gedacht. Die weiteren Zeilen schildern die haruspicatio in allen Details; erkennbar ist dies trotz der Lücken an den erhaltenen Wörtern SPLANCHNA REDDER/E in Z. I70 und splan]CHNA in Z. I7I. Zur haruspicatio wurde ein Gebet gesprochen, wie die Worte i]TA PRE[catus est (Z. I70) belegen. ${ }^{52}$

In Z. I7 I ist die Schilderung des Opfers von zwei Ochsen mit anschließender haruspicatio abgeschlossen. Nicht erhalten sind die auf das Opfer folgenden Riten von sellisternia und ludi, deren mögliche Schilderung in den Zeilen I 7 I und I 72 in keiner Weise der Schilderung dieser Riten nach dem Moiren-Opfer entspricht, wo ihnen mit knapp ro Zeilen, von denen allerdings fast nichts erhalten ist, eine ausführliche Darstellung zukam. Möglicherweise wurde die Darstellung dieser wiederholten Riten wie die Gebete abgekürzt.

Das detailliert beschriebene Opfer für Iuppiter kann trotz der vielen Lücken im Text so gegliedert werden:

Z. I55-I 57 Vorbereitungen (provisorischer Altar, Kleiderwechsel)

Z. I57-I 58 Opfergebet für beide Ochsen

Z. I58-I59 Vorbereitung (immolatio) für die Opferung des ersten Ochsen

Z. I 59-160 Bitte um Annahme des ersten Ochsen

Z. I60-I63 Vorbereitung (immolatio) für die Opferung des zweiten Ochsen

Z. I63-I64 Opfergebet für den zweiten Ochsen

Z. I64-165 mox haruspicatio - Vorbereitungen für die haruspicatio(?)

Z. i65 Abschneiden der Stirnlocke eines Ochsen, Herbeischaffen eines Kranzes

Z. I65-ı66 Gebet und Opfer von Kränzen (für Iuppiter ?)

Z. I66-I67 dreifaches Besprengen des Altars mit Wasser (oder Blut?)

Z. 167-168 Anweisung zum Wasserholen und Reinigungen

Z. I69-172 haruspicatio mit Gebet

I72-I79 Trotz kaum lesbaren Textes kann mit Sicherheit behauptet werden, dass hier die Riten um das Opfer für die Ilithyien wiedergegeben werden. Diesen wurde in der zweiten Nacht, vom I. auf den 2. Juni, geopfert. Die wenigen erhaltenen Buchstaben am rechten Rand lassen eine Rekonstruktion des Gebetstextes zu. ${ }^{53}$ Alle Angaben über die Beteiligten, das

52 Scheid (2005) I06f. macht auf den Gebrauch des griechischen Wortes splanchna anstelle von exta aufmerksam. Dies könnte auf eine Opferteilung nach ritus Graecus hinweisen, wo die Eingeweide von den Opfernden verspeist wurden. Scheid vermutet beim Iuppiter-Opfer eine Art Verdoppelung des Opferritus, wobei ein eindeutig römischer Opferritus mit Elementen nach dem ritus Graecus erweitert wurde. 
Datum und den Ort sind nicht erhalten, lassen sich aber aus den Angaben der augusteischen Inschrift zwar nicht ergänzen, aber erschließen. Auch dieses Opfer wurde von Septimius Severus vollzogen, wahrscheinlich wieder zusammen mit Caracalla, der das Gebet vorsprach. Als Opfergabe erhielten die Ilithyien von drei verschiedenen Arten Gebäck je neun Stücke. Es sind dieselben Opfergaben, die am dritten Tag Apollo und Diana erhalten werden. ${ }^{54}$ Die Darstellung der Riten der zweiten Nacht der Säkularspiele erstreckt sich bis Z. 179. Nach dem Gebet (Z. I72f.) folgt die Schilderung der Riten, die immer an die Opfer anschließen: ludi saeculares im provisorisch errichteten Theater im Tarentum und sellisternia der Matronen. Die Zeilen 177 und 178 enthielten weitere Angaben über Handlungen nach dem Opfer; AQVA SPARSA kann hier nicht wie in Z. 167 im Rahmen einer haruspicatio vollzogen worden sein, da kein Tier geopfert wurde.

Nach den ludi saeculares bewegte sich der Zug der Feiernden auf der üblichen Strecke zum Kapitol, um dort am frühen Morgen die Opfer des zweiten Tages zu feiern. ${ }^{s}$

Wieder erwähnt die Inschrift den Kleiderwechsel für diese Prozession mit PRAETEXTIS SVMPTIS ET CORONIS [accitis (Z. I79). Anschließend wird mit Angabe der führenden Matrone Iulia Domna, die mit ihrem Ehrentitel Mater castrorum und als Ehefrau des Kaisers erwähnt wird, die Abhaltung der sellisternia verzeichnet; dazu sind die Matronen in der Prozession vom Tarentum auf das Kapitol gezogen.

179-I83 Die Ereignisse des zweiten Tages der ludi saeculares, des 2. Juni, bildeten den Höhepunkt der Säkularfeier. Sie nehmen in beiden Inschriften viel Raum ein. ${ }^{56}$ Das Iuno-Opfer wird auf dem Kapitol abgehalten. Die Quindecimviri halten ein zusätzliches sacrificium epulare ab, die Matronen führen eine supplicatio durch, anschließend finden wieder Spiele im Tarentum statt und sellisternia der Matronen auf dem Kapitol. Die severische Inschrift verzeichnet außerdem über i7 Zeilen alle Namen der Matronen und die ihrer Ehemänner.

Der Darstellung des Opfers einer weißen Färse geht eine ausführliche Schilderung der Handlungen vor dem Opfer voraus: Die praefatio wird

54 Zum Ilithyien-Opfer siehe den Sachkommentar zu Act. Aug. i I5-I i 8. Apollo-/Diana-Opfer Act. Sev. 228-234. Scheid (2005) Iorf. warnt davor, fleischlose Opfer von vornherein für weniger wichtig zu halten. Die Zubereitung von Gebäck wird bei den Arvalbrüdern während des Opferkults selbst vollzogen; allerdings gibt es auch Gegenbeispiele, sodass für den Fall des Opfers von Gebäck für die Ilithyien offenbleibt, wie sich der Opferritus genau abgespielt hat.

$55 \mathrm{Zu}$ der Prozessionsstrecke der ludi saeculares siehe Kap. A.7, S. 214, und zu den Abweichungen der severischen Spiele Kap. B.6, S. $473 \mathrm{f}$.

56 Act. Aug. I19-133; Act. Sev. 179-206. 
von Severus mit Caracalla vollzogen, Caracalla spricht das dazugehörige Gebet vor (Z. ı 80). Severus vollzieht das Opfer allein, gemäß der neuen Lesart FECI $\left[\right.$ t $^{57}$ Anschließend legen die Opfernden die praetexta und den Kranz ab, um in der tunica fimbriata und ohne Kopfbedeckung nach ritus Graecus das Opfer durchzuführen (Z. I 80). Anwesend sind neben Septimius Severus und Caracalla auch Geta, Plautianus und die übrigen Quindecimviri (Z. I8I). Mit beiden Händen (Z. I8०f.) hält Severus das Opfermesser, mit dem bei der immolatio über den Rücken des Opfertieres gestrichen wurde. Ebenso ist die Opferschale mit Wein erwähnt, mit welchem die Stirn des Opfertiers besprengt wurde. Das Opfergebet wird bei diesem Opfer von Geta vorgesprochen - RETINENTE PR\{A\}ECATIONE[[m Geta Caes.]]. Anschließend opfert Septimius Severus die junge Kuh nach GRAECO ACHIVO RIT]u, also ohne Kopfbedeckung (Z. I 8 I). ${ }^{8}$ Die Bekleidung des Opfernden in tunica fimbriata signalisiert bereits, dass der Opferritus nicht mit typisch römischer Bekleidung in toga praetexta durchgeführt wurde. ${ }^{59}$

Das Opfergebet ist nach der zweiten Bitte wie üblich abgekürzt mit cetera ut supra.

I $82 \mathrm{f}$. Nur bei der Schilderung des Opfers für Iuno sind Angaben über die Aktivitäten direkt nach dem Opfer überliefert. Ein zusätzliches sacrificium epulare für Iuppiter wurde abgehalten und im Rahmen dieses $\mathrm{Op}$ fers (Z. I 83: religio]NIS CAVSA EPVL[ati sunt.) ein Bankett gefeiert, denn erst durch ein gemeinsames Mahl mit den Göttern erfüllte das Opfer an Iuno seine Funktion. Genaue Aussagen über den Ort, den Ablauf, das Opfer, das verzehrte Mahl lassen sich nicht machen. Als Teilnehmer des Banketts sind die beiden Augusti, Septimius Severus und Caracalla, sowie zwei der Quindecimviri, Fabius Magnus und Aiacius Modestus, aufgeführt. Trotz des lückenhaften Textes ist klar, dass weitere Beteiligte nicht möglich sind. Sie tragen immer noch die tunica fimbri$a t a,{ }^{60}$ wodurch dieses Opfermahl zu den Handlungen nach ritus Graecus zu gehören schien und als Teil der Riten des zweiten Tages gesehen wurde.

57 Alle früheren Bearbeiter der Inschrift haben FECE[runt gelesen und waren somit gezwungen, ein weiteres Subjekt neben Severus zu ergänzen.

58 Auch das Iuno-Opfer der augusteischen Säkularspiele wurde nach Achivo ritu vollzogen. Trotz der Einwände von Lipka (2009) I 52 n. I6 bleibe ich bei meiner Textergänzung von Act. Aug. I I 9 .

59 Nach Scheid (2005) rogf. zeigen gerade die Opfer der Säkularspiele, dass ein Opfer nach ritus Graecus nicht das Befolgen eines vollkommen anderen Ritus bedeutete, sondern die Erweiterung des üblichen römischen Ritus um gewisse fremde Elemente.

60 Der Kleiderwechsel zur toga praetexta kann erst vor der supplicatio (Z. I 83) ergänzt werden. 
Die Riten des zweiten Tages hatten bereits in der Nacht vom I. auf den 2. Juni begonnen. Nach dem nächtlichen Opfer für die Ilithyien und den ludi saeculares im Tarentum waren Septimius Severus, Caracalla, Geta, Plautianus und die übrigen Quindecimviri auf das Kapitol gezogen, um im Morgengrauen das Iuno-Opfer zu vollziehen. Die Matronen hatten auf dem Kapitol nach dem nächtlichen Opfer ihre sellisternia gefeiert (Z. 179). Es gibt keine vergleichbare Schilderung eines solchen Banketts nach den Opfern des I. oder 3. Juni. Es ist aber dennoch anzunehmen, dass ein solches immer nach einem Opfer am Tage bei den Säkularspielen stattgefunden hat. Anzumerken ist jedoch, dass während dieses Banketts ein weiteres Opfer vollzogen wurde. ${ }^{61}$

I83-I88 In Z. I 83 wird wieder die toga praetexta übergestreift. Geta, Plautianus und die übrigen Quindecimviri treten zu Septimius Severus und Caracalla vor die cella des Iuno-Tempels, wo bereits Iulia Domna und rog Matronen versammelt sind. Auch die zwei Vestalinnen Numisia Maximilla und Terentia Flavola sind anwesend. Septimius Severus spricht den i Io Matronen das Supplikationsgebet vor, welches die Matronen kniend nachsprechen. ${ }^{62}$ Als verheiratete Frauen bitten sie Iuno Regina (Z. I 85 f. und I88) in der ersten Person Plural mit denselben Bitten wie in den Opfergebeten um Wohlergehen und Stärke für das römische Volke, seine Legionen, die Quindecimviri, sich selbst, ihr Haus und dessen Bewohner. ${ }^{63}$ Mit dieser Supplikation der Matronen ist ein Höhepunkt der Säkularspiele erreicht. Wie wichtig dieser Auftritt im Rahmen der ludi saeculares war, wird an den folgenden Zeilen deutlich, in denen jede der I09 Matronen mit ihrem Ehemann namentlich erwähnt ist.

I 88-205 Trotz starker Fragmentierung der Namenslisten der Matronen und ihrer Ehemänner können zahlreiche Beziehungen zwischen allen Beteiligten an den ludi saeculares hergestellt werden. ${ }^{64}$

205f. Den Abschluss der Riten des zweiten Tages bildeten wie bereits am Tag zuvor sellisternia der Matronen für Iuno und Diana auf dem Kapitol, wohl auch vor dem Iuno-Tempel, wo sie gerade zuvor das Supplikationsgebet gesprochen hatten.

6I Vössing (2004) 53-59 beschäftigt sich mit der Frage, an welcher Stelle eines Banketts das Opfer vollzogen wurde, und kommt zu dem Schluss, dass es nach der Hauptspeise, aber vor dem Gelage, also bevor die Tische abgeräumt wurden, dargebracht wurde. Im Fall des hier beschriebenen Banketts, das während bedeutender religiöser Zeremonien eingeschoben wurde, können aber durchaus andere Regeln gegolten haben. Meine Ergänzung INTER [cenam sac]RIFICAVERVNT stützt sich auf Angaben der Arvalakten, siehe den Kommentar zur Textkonstitution (Kap. B.3) zu Z. I 83, S. 355 .

62 Act. Sev. i85 NVPT[ae genibus nixae] und Act. Sev. I 87 NVPTAE GENIB[us nixae.

$63 \mathrm{Zu}$ dem Akt der Supplikation und dem Gebet siehe den Sachkommentar zu Act. Aug. I $23 \mathrm{f}$.

64 Für detailliertere Angaben zur Prosopografie siehe Kap. B.5, S. 432 ff. 
206-208 Bevor die Feiernden ins Tarentum aufbrechen, um noch einmal ludi saeculares in dem provisorischen Theater anzuschauen, ist ein Bericht über die Auslosung des Vorsitzes bei den ludi honorarii eingeschoben. Wie bei der Auslosung über die Dreiergruppen auf den Tribünen zur Verteilung der suffimenta (Z.68-7I) wird die Auslosung mit Würfeln durchgeführt, die in eine Urne geworfen und anschließend gezogen werden. Es folgt eine Liste der anwesenden Quindecimviri, die nicht vollständig erhalten ist. Vierzehn Namen sind lesbar, der Raum genügt nicht, um wie in den Zeilen 65-68 das gesamte Kollegium aufzuführen. Zwölf dieser Namen werden in den Zeilen 269-272 nochmals erwähnt, wo sie den Vorsitz an verschiedenen Spielstätten der ludi honorarii innnehaben.

208f. Hier wird die Prozession vom Kapitol ins Tarentum geschildert, wo wieder ludi saeculares aufgeführt werden.

209-222 Für I 3 Zeilen ist die protokollarische Schilderung der Säkularfeier unterbrochen, um ein Edikt zu zitieren, das zu diesem Zeitpunkt, nach den Riten des zweiten Tages, erlassen wurde (Z. 209: ET E[odem die). Es geht darin um die Ankündigung zusätzlicher Spiele, sogenannter ludi bonorarii. ${ }^{65}$ Nach der üblichen Edikteinleitung mit Titulaturen und Filiationen von Septimius Severus, Caracalla und Geta (Z. 209-2 I2) werden Spiele für weitere sieben Tage verkündet (Z. 2 I2-2 I4). Das Programm dieser sieben Tage sieht Folgendes vor:

\begin{tabular}{|c|c|c|}
\hline 4., 5., 6. Juni & ludi scaenici & $\begin{array}{l}\text { THEATRIS TRIBVS, LIGNEO, } \\
\text { POMPEIANO, ODI[O (Z.213) }\end{array}$ \\
\hline 7.-IO. Juni & ludi circenses & IN CIRCO MAXIMO (Z. 214) \\
\hline
\end{tabular}

Die folgenden Zeilen des Edikts geben die verschiedenen Rennen mit den vorgesehenen Preisen für die ersten drei der Wagenlenker bekannt. Die beiden ersten Rennen bestreiten Viergespanne, das dritte Zweispänner. Ein viertes Rennen ist für Kunstreiter und Wettläufer (Z. 2I4f.), ein fünftes für $Z$ weispänner vorgesehen, ein sechstes für Kunstreiter, und das siebente und letzte Rennen findet wieder mit Viergespannen statt (Z. 2 I6f.). Nach sieben Rennen im Circus Maximus werden Tierhetzen mit 700 wilden Tieren gegeben, deren beeindruckende Vielfalt in Z. 2 I 9 aufgeführt ist. ${ }^{66}$ Die erwähnten Tiere stammten wahrscheinlich alle aus Nordafrika, womit zusätzlich auf die munificentia des Kaisers hingewiesen wurde. Weil für diese Tierhetzen Sicherheitsmaßnahmen für die $\mathrm{Zu}-$ schauer getroffen werden mussten, wird die Bevölkerung angewiesen,

65 Auch die augusteische Inschrift enthält ein entsprechendes Edikt mit der Ankündigung zusätzlicher Spiele. Vgl. Act. Aug. I $55^{-1} 58$ und I62f.

66 Vgl. Cass. Dio 77, I, I ff. Dort wird von Spielen berichtet, die zum ersten Decennium der Regierung unter Septimius Severus im Jahr 203 abgehalten und mit ähnlichen Tieren bestritten wurden. 
strikt den Anordnungen des Sicherheitspersonals zu folgen (2I 8 f.). Das Edikt schließt mit der Einteilung der Pantomimen Pylades, Apolaustus und Marcus während der ludi honorarii. Diese sind vom 4. bis 6. Juni in drei verschiedenen Theatern im Marsfeld nach einem Rotationsplan eingesetzt. ${ }^{67} \mathrm{Im}$ Gegensatz zu den augusteischen ludi honorarii finden im Jahr 204 n. Chr. keine Aufführungen im Marcellus-Theater statt, weil es zu diesem Zeitpunkt wohl in schlechtem Zustand war. ${ }^{68}$ Stattdessen ist das Odeum des Domitian neben dem provisorischen Theater im Tarentum und dem Theater des Pompeius vorgesehen.

Die severischen ludi honorarii grenzen sich klar von den ludi sollemnes ab (per]ACTIS LUDIS [sollemnibus Z. 212). Mit ludi sollemnes sind Spiele gemeint, die im römischen Festkalender festgelegt sind und nicht zusätzlich angeordnet werden müssen. Auch hier haben die Organisatoren der severischen ludi saeculares aus der augusteischen Inschrift gelernt und haben den Verlauf der Feierlichkeiten nach den ludi saeculares in einem einzigen Edikt festgelegt, während bei den augusteischen ludi saeculares zuerst ein Edikt über die ludi scaenici erlassen wurde und nach deren Ablauf ein weiteres über eine venatio und Wagenrennen. ${ }^{69}$

222-228 Nach dem Edikttext setzt die Inschrift die Schilderung des Ablaufs der ludi saeculares fort. Es folgen die Riten der dritten Nacht für Terra Mater. ${ }^{70}$ Dazu sind die vier Mitglieder der kaiserlichen Familie wieder in toga praetexta und mit Kränzen vom Palatin in feierlichem Zug zum Tarentum gezogen (Z. 223). Dort hat Septimius Severus der Terra Mater auf einem dritten hölzernen Altar, ${ }^{71}$ der wie die beiden anderen provisorisch errichtet worden war, in toga praetexta die praefatio vollzogen (Z. 224); das Gebet dazu sprach Caracalla vor. Anschließend wurden Toga und Kranz abgelegt, um die tunica fimbriata anzuziehen. Durch Opfersklaven des Kollegiums ließen sich die vier Mitglieder der kaiserlichen Familie die Hände reinigen, Geta, Plautianus und die Quindecimviri standen dabei (Z. 224). Geta wird mit dem Vorsprechen des Opfergebets in den Opferritus einbezogen (RETENENTE PRECATIONE[[m

67 Zur Identifikation der drei Pantomimen vgl. Leppin (I992): Pylades 287 (Pylades VI); Apolaustus 206 (Apolaustus IV), Marcus 258.

68 Vgl. Anm. 89.

69 Die ludi scaenici sind in der augusteischen Inschrift gemäß ihrem Charakter mit ludi Latini, ludi Graeci thymelici und ludi Graeci astici bezeichnet. Siehe den Sachkommentar zu Act. Aug. I55-I 58 .

70 Vgl. den Sachkommentar zu Act. Aug. I34-I37.

7I Dass für die im Heiligtum des Dis Pater und der Proserpina verehrten Göttinnen der Säkularspiele eigene Altäre provisorisch errichtet wurden, dokumentiert ein Gastrecht, dass sich auf einen Ort bezieht, aber nicht auf einen gemeinsam benutzten Altar. Nach Zosimus 2, 5, 2 wurde während der Säkularspiele auch Dis Pater und Proserpina geopfert. Siehe Anm. 23 I im Sachkommentar zu Act. Aug. 9of. (S. I 8 f.). 
Geta C[aes.]] Z. 225). Mit beiden Händen hat Severus das Opfermesser und eine Opferschale mit Wein gehalten und die immolatio vollzogen, um darauf das Opfer einer trächtigen Sau nach GRAECO A(chivo) $R$ (itu) durchführen zu lassen (Z. 225). Das abgekürzte Gebet ist sehr gut erhalten und wie die übrigen Gebete nach der zweiten Bitte abgekürzt (Z. 225227). Ausdrücklich verweist die Inschrift darauf, dass dieses Opfer e]ODEM MODO wie die Opfer für die Moiren in der ersten Nacht der Spiele durchgeführt wurde, nämlich nach ritus Graecus und holokaust. Die nächtlichen Riten werden fortgesetzt mit den ludi saeculares in dem provisorischen Holztheater. Dazu wurde die tunica fimbriata wieder abgelegt und die römische Kleidung mit Kranz angezogen (Z. 227).

Die sellisternia für Iuno und Diana von Iulia Domna und Io9 Matronen schlossen die nächtlichen Riten ab (Z. 228). Die sellisternia fanden wieder auf dem Kapitol in der cella des Iuno-Tempels oder davor statt.

228-233 Die ab Z. 228 geschilderten Riten des dritten Tages werden mit einer auffälligen Angabe des Datums eingeleitet, die trotz des begrenzten Raums im unteren Teil der Inschrift von zwei sehr langen vacat umgeben ist.

Am 3. Juni wurde das letzte Opfer der Säkularspiele vollzogen. Es galt Apollo und Diana und bestand aus je drei Exemplaren unterschiedlicher Gebäcke. Es wurde gleich wie das Opfer der zweiten Nacht für die Ilithyien durchgeführt. Septimius Severus, Caracalla, Geta und Plautianus mit den Quindecimviri waren in toga praetexta und bekränzt zum Tempel des Apollo auf dem Palatin gezogen (Z. 229). Dort befand sich ein hölzerner provisorisch errichteter Altar (Z. 229f.). Der Ablauf des Opfers ist wie üblich: praefatio mit dem Vorsprechen des Gebets durch Caracalla, Z. 230), darauf das Opfergebet, das in diesem Fall Septimius Severus selbst liest (HAC PREC[a]TION[e quam le]GIT IPSE). ${ }^{72}$ Das Gebet (Z. 23 If.) ist wieder wie üblich abgekürzt. Für Diana wurden dieselben Opfergaben auf dieselbe Weise geopfert (Z. 233).73

Es fällt auf, dass in dem gut erhaltenen Text keine Angaben über einen Kleiderwechsel von toga praetexta $\mathrm{zu}$ tunica fimbriata $\mathrm{zu}$ finden sind. Das könnte bedeuten, dass die Opfer für Apollo und Diana nicht nach Graecus ritus vollzogen wurden. Das anschließende Singen des carmen saeculare ist ein typisch römischer Brauch; dies unterstützt den Eindruck, dass die Riten des dritten Tages vollständig nach römischer Sitte durchgeführt wurden. ${ }^{7}$

72 Es bleibt offen, ob auch bei einem fleischlosen Opfer eine immolatio stattfand; erwähnt ist sie weder in den Act. Aug. noch in den Act. Sev. Vgl. Scheid (2007) Ior.

73 Zum Ablauf des Gebäckopfers vgl. den Sachkommentar zu Act. Aug. I I 5-I i 8, S. I 46; zum Apollo- und Diana-Opfer den Sachkommentar zu Act. Aug. I39-I46, S. I 53 f.

74 Act. Aug. I39-I46 erwähnt ebenfalls keinen Graecus ritus. 
Nach dem Opfer für Apollo und Diana haben die Opfernden sich noch einmal zum Tarentum begeben, um dort im Holztheater neben dem Tiber die ludi saeculares anzuschauen.

Darauf wurde auf dem Palatin von 27 Knaben und 27 Mädchen das carmen saeculare vorgetragen. Diese Kinder aus vornehmen Familien ${ }^{75}$ mussten noch beide Elternteile haben (patrimi et matrimi - Z. 235), eine im römischen Kult übliche Forderung, die die Unversehrtheit und Reinheit der Kinder ausdrückte. Das severische carmen saeculare wurde wie das von Horaz eigens für diesen Anlass komponiert und war ursprünglich im Wortlaut wiedergegeben, ist aber nur sehr fragmentarisch erhalten. Trotz der Bemühungen zahlreicher Latinisten lässt sich dieser Text nicht mehr rekonstruieren. Einzig über die Länge kann man sagen, dass es in seinem Umfang dem des horazischen carmen saeculare entsprochen hat. ${ }^{6}$ Es wurde mit Instrumentalbegleitung vorgetragen (TIBICINIBVS Z. 236) und ist an Apollo und Diana-Lucina (Z. 239) gerichtet. An einigen Worten lässt sich ermessen, dass sein Inhalt sich um die Größe Roms und seines Volkes drehte: PRO GENTIS HON[o]RE (Z. 237), SVPERBO DE GREGE NATORVIm (Z. 238). Der von Septimius Severus verehrte Bacchus fand Erwähnung (Z. 243), ${ }^{77}$ wahrscheinlich auch seine siegreichen Feldzüge (Z. $245 \mathrm{f}$.). Wie das carmen saeculare von Horaz verherrlichte es das Fortbestehen der Größe des Imperium Romanum durch eine gesicherte Nachkommenschaft. Diese war beim Singen des carmen saeculare nicht nur durch die beiden Chöre sichtbar und präsent, sondern bei allen Opfern durch Septimius Severus mit seinen beiden Söhnen und dem Schwiegervater des älteren Sohnes, Plautianus. Caracalla und Plautilla hatten zum Zeitpunkt der ludi saeculares möglicherweise bereits ein Kind. ${ }^{78}$ So repräsentierte Septimius Severus mit dem Auftreten seiner Familie den Fortbestand Roms unter der severischen Dynastie.

75 Außer einem sind alle Knaben senatorischer Herkunft; die Mädchen stammen alle aus senatorischen Familien. Vgl. Kap. B.5, S. 426.

76 Das horazische carmen saeculare umfasst ig Strophen mit durchschnittlich Io० Buchstaben pro Strophe, das severische carmen saeculare erstreckt sich über gut i I Zeilen mit durchschnittlich i 80 Buchstaben pro Zeile.

77 Die Nennung von Bacchus als einem der Di Patrii des Septimius Severus hat zu Diskussionen über die Rolle von Liber Pater und Hercules an den ludi saeculares geführt. Lichtenberger (20I I) $272 \mathrm{f}$. weist darauf hin, dass neben der Nennung von Bacchus im carmen saeculare die Berücksichtigung dieser Götter an den Säkularspielen durch is Münzprägungen unterstrichen wurde, die sich auf die Säkularspiele beziehen und Abbildungen dieser Götter tragen.

78 Gagé (1934) 35f. weist auf eine Darstellung der pietas Augustorum auf Münzen der Plautilla hin. Das Motiv des Haltens eines Kindes könnte auf eine Geburt im Kaiserhaus anspielen. Ebenso Lichtenberger (20I I) 275, der einen aureus der Plautilla zeigt (Abb. I 57), auf dessen Rückseite eine Iuno Lucina thront. 
247-266 Hier beginnen die Riten, die den Abschluss der ludi saeculares einleiten. Wir haben in den Zeilen i 18-i 2 I gesehen, dass der Auftakt der ludi saeculares aus einer Prozession von der Stadt zum Tarentum bestand. Ebenso stand am Ende der Spiele eine grandiose Prozession. Sie führte vom Palatin, wo gerade das carmen saeculare gesungen worden war, über die via sacra durch den Severus-Bogen zum Kapitol (Z. 249) und dürfte damit denselben Weg genommen haben, wie die Prozession zum Beginn der Spiele. Vom Kapitol wurde die Prozession bis ins Tarentum fortgesetzt, wo die ludi saeculares ihren Abschluß fanden.

Allerdings ist die Abschlußprozession von zahlreichen Anreicherungen und Verzögerungen geprägt. Ausdrücklich werden Flötenspieler, Hornund andere Blechbläser erwähnt: TIBI[cinum, cornic]INVM, AENEATORVM ET TVBICIN[u]M TRANSLATVM (Z. 247). Daneben traten zahlreiche Teilnehmer der folgenden ludi honorarii auf, um die Vorfreude darauf zu wecken. Zu diesen gehörten Eseltreiber, Knappen, pantomimische Tänzer, Viergespanne, Zweigespanne, Kunstreiter und Läufer (Z. 248). Auch die Ordnungshüter und das zu beiden Seiten des Zuges stehende Volk haben Eingang in den Text der Inschrift gefunden (Z. 249). Es folgen Angaben über die Route der Prozession. Ausgangspunkt ist der Palatin, wo nach den Opfern des letzten Tages für Apollo und Diana das carmen saeculare gesungen worden war. Dort formierte sich der Zug und bewegte sich vom Vorplatz des Apollo-Tempels zuerst nach Osten. Der Platz vor dem 203 n. Chr. eingeweihten Septizodium bot genügend Raum, dass sich mehr Teilnehmer dem Prozessionszug anschließen konnten; außerdem müssen große Menschenmengen den Zug verfolgt haben (i]NTERCEDE[nte popul]O Z. 249)..$^{79}$ Die drei Severer bildeten vor den Sängern und Sängerinnen des carmen saeculare die Spitze des Zuges (PRAECE[debant Z. 250). Wahrscheinlich waren auch das Kollegium der Quindecimviri und andere Würdenträger in dieser ersten Gruppe, denn in Z. 250 ist beim Halt des Zuges auf dem Kapitol auch Plautianus erwähnt. Dort wurde das carmen saeculare - genauso wie auf dem Palatin noch einmal vorgetragen. Diesmal gibt die Inschrift zusätzliche Informationen über die Aufführungspraxis: Die Kinder hielten sich an den Händen und die Mädchen tanzten eine Art Reigen (Z. 25 I). Auch hier wurde noch einmal ein Opfer ausgerichtet, über welches keine weiteren Angaben gemacht werden (PERFE[cto] SACRIFICIO Z. 25I). Diese Stellung des carmen saeculare zwischen zwei Opfern bestätigt seine Bedeutung als Teil des Rituals.

Die Knaben und Mädchen wurden von den beiden Augusti, Septimius Severus und Caracalla, mit Geschenken bedacht. Die Knaben erhielten

79 Zur Route der Prozession siehe Kap. B.6, S. 459 ff. 
neun silberne Schalen, die Mädchen drei Stücke Seidenstoff und festlichen Schmuck (PRA[etext(am)] SOLLEMNEM Z. 252). In einem Vorgriff auf das künftige Geschehen wird mitgeteilt, dass auch die Knaben, die später am lusus Troiae beteiligt sind, neun silberne Schalen als Geschenk erhielten, wobei möglicherweise einige ältere der Chorknaben auch am lusus Troiae teilgenommen haben. ${ }^{80}$ Anschließend traten die Kinder ab und nahmen nicht mehr an der Fortsetzung der Prozession teil (SE RECEPER [unt Z. 252).

Nach dem Halt auf dem Kapitol bewegte sich der Zug weiter zum Tarentum, um zum letzten Mal ludi saeculares in dem Holztheater am Tiber anzuschauen und damit die Säkularspiele abzuschließen ( $A D L V D O S$ SAECVLARES CONSVMMANDO[s] IN THEA[tro lign]EO Z. 253). Als Teilnehmer an der Spitze des Zuges sind wieder die beiden Augusti, Geta, Plautianus und die Quindecimviri genannt (Z. 252 f.). Die Ausführungen von Abaecherli Boyce haben diesen Teil der Prozession präzisiert. ${ }^{81}$ Es handelte sich um eine pompa sacrificalis, in deren Zusammenhang wohl auch das Opfer auf dem Kapitol gesehen werden muss. Der Zug bewegte sich auf der üblichen Route vom Kapitol über das Marcellus Theater und die porticus Octaviae ${ }^{82}$ zu der Rennbahn, die wie das Holztheater vorübergehend entlang dem Tiber eingerichtet war. ${ }^{8_{3}}$ Es gab jedoch vorher noch einen Halt und eine Runde in diesem Zirkus (in cir]CO TEMPORAL[i] CIRCV[mdu]CTA EST Z. 253). Ein Teil des Zuges blieb bereits im Zirkus und zog nicht weiter in das theatrum ligneum, das für die begleitende Schar sicher zu klein war. Für die Teilnahme der letzten ludi saeculares im daneben liegenden Holztheater sind nur die beiden Augusti erwähnt (Z. 253 f.). Diese haben erst nach den ludi saeculares im Holztheater mit Palmenzweigen und Elfenbein-Zeptern den Zirkus aufgesucht und dort über den Startboxen (supra cJARCERES Z. 254) Platz genommen. Die anschließenden Spiele bestanden aus Rennen mit Viergespannen, Zweigespannen, Kunstreitern und Wettläufern. Diese Zirkusspiele wurden offenbar auf andere Art abgehalten als die Spiele an den folgenden Tagen im Circus Maximus; wahrscheinlich war die Rennbahn kürzer und die Rennen deswegen weniger spektakulär. Die Inschrift erwähnt ausdrücklich, dass es sich um Veranstaltungen ANTIQVI MORIS (Z. 256) handelt. Dazu gehörten wohl auch die in den folgenden Zeilen erwähnten und nicht mehr verständlichen Hinweise auf $p$ ?]VLENTA [P]ETENTIVM (Z. 256) und SOLEIS FETASIIS (Z. 257), womit viel-

80 Vgl. Kap. B.5, S. 438 .

8 I Abaecherli Boyce (I94I) $3 \mathrm{f}$.

82 Act. Sev. I 8 f.

83 Siehe Kap. A.7 mit einem Plan S. 203. 
leicht auf eine allgemeine Essensausgabe und eine bestimmte Fußbekleidung hingewiesen wird. Die Spiele wurden auch am Nachmittag fortgesetzt mit derselben Art von Veranstaltungen (Z. 257). Am Ende der Spiele erhielten die Akteure als Belohnung die Früchte, die das Volk vor dem Beginn der ludi saeculares in der acceptio frugum zusammengetragen hatte (Z. 258 f.). Die noch verbleibenden Früchte erhielten die Aufseher in einer zweiten Verteilaktion. All diese Handlungen und die Positionierung der Zirkusspiele im Festprogramm vor den sellisternia der Matronen machen deutlich, dass die Zirkuspiele im Tarentum noch als Teil der ludi saeculares aufgefasst wurden. Hier wird der im Orakel formulierten Forderung nach Freude und Fröhlichkeit entsprochen. ${ }^{8}$ Die ludi honorarii, die erst nach dem offiziellen Abschluss der Säkularspiele stattfanden, stellten eine freiwillige Steigerung dieses Vergnügungsprogramms dar.

Nach Abschluss der Spiele am Nachmittag (Z. 259) hielten die I Io Matronen zum letzten Mal ihre sellisternia ab. Diese fanden zwar wie an den zwei Tagen vorher statt (SICVT PRAEC[eden]TI BIDVO Z. 260). Die Inschrift liefert in diesem Fall aber zusätzliche Angaben: Die Matronen haben eigene Opfer von jungen Säuen verrichtet (PORCILIAS IMMOLAVERVNT Z. 260), gespeist, getanzt und rhythmisch mit den Füßen gestampft (CENA[verunt et antr]VAV[erunt Z. 260). Auch wenn die Ergänzung von antr]VAV[erunt durch Pighi umstritten ist, zeigt diese Präzisierung das Ausmaß der Aktivitäten der Matronen an den ludi saeculares. ${ }^{85}$ Es ist nicht klar, ob die Inschrift diese letzten sellisternia ausführlicher dokumentiert als die vorangegangenen, zumal an dieser Stelle den Redakteuren bewusst sein musste, dass die Fläche auf der Vorderseite des Steins nicht ausreichen wird und weitere Angaben auf der seitlichen Fläche gemacht werden müssen.

Als letzte Ehrung der beteiligten Kinder sind deren Namen verzeichnet. Damit liefert die severische Inschrift den einmaligen Hinweis auf die Namen von Kindern der Oberschicht, die als Angehörige anderer namentlich genannter Protagonisten der severischen Säkularspiele identifiziert werden können. ${ }^{86}$

266-272 Das vacat mit dem folgenden Datum in Z. 266 ([Pr.] NON. IVN.) verweist darauf, dass nun in den drei untersten Zeilen auf der Frontseite der Inschrift mit der Protokollierung der Ereignisse nach den eigentlichen ludi saeculares begonnen wird. Am 4. Juni begannen ohne dazwischen liegenden Ruhetag die ludi honorarii. Dazu begaben sich die vier Mitglieder der kaiserlichen Familie und das Kollegium der Quindecimviri zu

84 Siehe Orakeltext V. 35, S. 24I.

85 Sterbenc Erker (2015) I 27 sieht in dieser Stelle neben anderen einen Hinweis auf eine eigene Opferpraxis von Frauen auch in der römischen Republik.

86 Vgl. Kap. B.5, S. $432 \mathrm{ff}$. 
den drei Spielstätten, wo jeweils unterschiedliche Unterhaltungsprogramme angeboten wurden: IN THEATRO LIGNEO (Z. 267) wurden ludi Latini aufgeführt, IN ODIO (Z. 267) ludi Graeci und IN THEATRO POMPEIAN[o (Z. 267) ludi scaenici. ${ }^{87}$ Damit übernimmt die severische Feier nicht die Vorgaben der augusteischen, an welcher der 4. Juni ein Ruhetag war. ${ }^{88}$ Auch die Orte, an welchen die augusteischen ludi bonorarii stattfanden, sind nicht dieselben: Statt des Marcellus-Theaters, das an den augusteischen Spielen benutzt wurde, wurde an den severischen Spielen das unter Domitian errichtete Odeum als Spielstätte genutzt. ${ }^{89}$ Die veränderte Topografie der Spielstätten bestimmte die Route der Prozession: Nachdem die beiden Augusti den Anfang der ludi Latini im Holztheater begangen hatten (LVD[is] LATIN[is comm]ISSIS Z. 267), begaben sie sich ins Odeum, wo sie ebenfalls nur den Anfang der ludi Graeci mitmachten. Anschließend ging der Zug weiter ins Theater des Pompeius, wo sie weitere ludi scaenici eröffneten. Von dort begaben sich die beiden Augusti in den provisorischen Zirkus am Tiberufer. Zusammen mit Geta setzten sie sich dort (Z. 268). Damit hatte ihre Prozession ein Ende gefunden. Im Zirkus verfolgten sie die dort veranstalteten Spiele bis zum Ende (PERSP[ect]AVERVNT Z. 268). Das Kollegium der Quindecimviri ist ebenfalls erwähnt, musste sich aber mit Stehplätzen begnügen: $(X V$ VIR(i) AVTEM TRIBVNALI PRAET[orio .5.] ST[eterunt.], Z. 268). Wahrscheinlich trafen die Quindecimviri erst nach und nach im Zirkus ein, denn fünfzehn ihrer Mitglieder hatten in den drei Theatern den Vorsitz bei den dort stattfindenden Spielen inne..$^{90}$

Mit diesen Angaben ist der Text auf der Vorderseite der Inschrift abgeschlossen; er wird fortlaufend auf der rechten Seite des Steines fortgesetzt.

87 Über den unterschiedlichen Charakter dieser Veranstaltungen vgl. den Sachkommentar zu Act. Aug. I55-I 58 .

88 Act. Aug. I 59: INTERMISSO DIE QVI FVIT P[rid. non. Iun.

89 Dass das Odeum unter Domitian ebenfalls im Hinblick auf seine ludi saeculares gebaut worden war, wird von Abaecherli Boyce (I94I) $47 \mathrm{f}$. in Erwägung gezogen. Sie weist darauf hin, dass möglicherweise der schlechte Zustand des Marcellus-Theaters seine Nutzung während der severischen ludi saeculares untersagte. Darin könnte später für Alexander Severus ein Grund gelegen haben, dieses Theater renovieren zu lassen. Gorrie (2002) 464 hält eine Verbindung des domitianischen Odeum mit den Säkularspielen von $88 \mathrm{n}$. Chr. ebenfalls für wahrscheinlich.

90 Abaecherli Boyce (2007) 45-47; die augusteische Inschrift nennt im Edikt der ludi honorarii (Act. Aug. I 57 f.) jeweils die Anfangszeit der Spiele in den drei Spielstätten. Da diese immer mit einer Stunde Abstand beginnen, schloss Abaecherli Boyce, dass Augustus nur die Eröffnung der Spiele mitmachte und dann zur nächsten Spielstätte weiterzog. Erst in der letzten Spielstätte, dem Marcellus-Theater, hat er die Spiele von Anfang bis Ende angeschaut. Für die severischen Spiele vermutet Abaecherli Boyce mit guten Gründen ein gleiches Vorgehen. 
Dort geben die ersten vier Zeilen an, welche Quindecimviri in welchem Theater den Vorsitz hatten.

in theatro ligneo Venidius Rufus cur.] ALV. TIBERIS, SAEVIN[ius Proc]VLVS, FVLVIUS FUVSC[us Grani]ANVS Q. AUG[gg.]| [Gargilius Antiquus, Ulpius Sote]R COS. DESIG., in Odio P[ompeius] RVSONIANVS MAGIST[er, Vetin]A MAMERTI[nus,] [Manilius Fuscus, Cassius Piu]S MARCELLINVS Q. D[es. in theatro Pompei $\quad \cdots$ | Antiu]S CRESCENS CALPVR[nianus,

$$
\begin{aligned}
& \text {-rnu]S PR., } \\
& \text { AIACIVS MODEST[us, } \\
& \text { Ofilius] MACEDO, } \\
& \text { NONIVS ARRI[us Mucia]NVS. }
\end{aligned}
$$

Diese Angaben entsprechen denen in Z. 209f., wo die Ergebnisse der Auslosung über den Vorsitz an den ludi honorarii angegeben sind. An drei Spielstätten sind je fünf Quindecimviri eingesetzt, wodurch - wie bei dem Einsatz von je drei Quindecimviri an fünf verschiedenen Stationen zur Verteilung der suffimenta - der Name des Kollegiums sinnvoll wird. Auch wenn es aus deutlich mehr Mitgliedern bestand, waren immer nur Is Mitglieder öffentlich sichtbar in Einsatz..$^{91}$

273-28 I Diese Zeilen enthalten das letzte Edikt der Inschrift. Mit der Edikteinleitung ist nicht nur auf der Vorderseite, sondern auch auf der Seite des Steinblockes auf Augenhöhe die volle Titulatur des Kaisers lesbar, die insgesamt fast sieben Zeilen des Texts einnimmt. Wegen des schlechten Zustands kann über den Inhalt des Edikts nur wenig gesagt werden. Es geht um den Verlauf der nächsten Tage (Id.] IVN. PROXIMAS Z. 279). An diesen Tagen sollten vielleicht noch zusätzliche Opfer ( $p J O R C V M$ Z. 279) abgehalten werden. ${ }^{92}$

Das Wort DISSIGNATOR (Platzanweiser, Z. 280) lässt vermuten, dass im Edikt die Organisation der weiteren Spiele im Circus Maximus bestimmt wurde. ${ }^{93}$ Über diese populären Spiele im Circus Maximus haben

9I Vgl. den Sachkommentar zu Act. Sev. 64-7I, S. $347 \mathrm{ff}$.

92 Die an das Edikt anschließenden, ebenfalls sehr schlecht erhaltenen Zeilen, legen nahe, dass weitere Opfer hier protokolliert sind.

93 Vgl. Act. Sev. 214: [dein die] VII IDVVM EARVNDEM CIRCENSIVM SPECTACVLA IN CIRCO MAXIMO DABIMVS. 
wir außer den Angaben des ersten Edikts der ludi honorarii (Z. 2I4-2 I9) keine weiteren Angaben. Während die ersten drei Tage der ludi honorarii mit den Aufführungen in den drei Theatern protokollarisch festgehalten sind (Z. 266-272), ist die Aufzeichnung der Veranstaltungen im Circus Maximus an den folgenden vier Tagen nicht mehr auszumachen. Es ist nicht klar, ob der schlechte Zustand des Texts dafür verantwortlich ist, oder ob dieser Teil für nicht aufzeichnungswürdig gehalten wurde.

282-298 Einige Wörter dieser Passage der Inschrift scheinen sich auf weitere Opferhandlungen zu beziehen. Die beiden Augusti mit Geta und Plautianus und den übrigen Quindecimviri sind vom Palatin (DE PALATIO Z. 282) gekommen. Auf einem pJVLNINAR Z. 283), einer Unterlage für ein Götterstandbild, wurde vielleicht ein Götterbild aufgestellt und ein Gebet gesprochen, wobei Geta allein direkt dabei stand ([[Geta [nob. Caes.]] ad]SISTENTE Z. 282). Im Gegensatz zu den Opfern der ludi saeculares sind hier Plautianus oder die Quindecimviri offenbar nicht erwähnt. ${ }^{94}$ Die Zeilen 284f. könnten Teile eines Gebets sein, wobei der zur Verfügung stehende Raum nicht genügt, um ein vollständiges Opfergebet aufzunehmen. Ganz klar verweist Z. 287 mit im]MOLAVIT IM [P. auf ein Opfer. Offenbar hat Septimius Severus ein weiteres Opfer einer Kuh für Iuno durchgeführt. Wem ein Schwein geopfert wurde, ist nicht klar. Ein Gegenstand der rituellen Praxis (ebjORNEOS Z. 288) ist erwähnt.

Im weiteren Text ist einzig mit Sicherheit der Name FVSCV[s Z. 295 lesbar und gehört wahrscheinlich dem Quindecimvir Fulvius Fuscus Granianus.

299-3 I7 Diese letzten zwei Fragemente der Inschrift sind ebenfalls stark zerstört. Für die Zeilen 299-305 geht aus den wenigen erhaltenen Buchstaben hervor, dass die oberen Zeilen die Namen von zwei Quindecimviri enthalten: Cassius Pius Marcellinus und Vetina Mamertinus (Z. $300 \mathrm{f}$.). Wieder sind Elfenbeinstab und Polsterkissen erwähnt, es geht auch hier um eine rituelle Handlung, die nicht mehr bestimmbar ist. Das Fragment der Zeilen 299-305 fehlt in der Aufstellung im Nationalmuseum in Rom.

Das letzte Fragment mit den Zeilen 306-317 enthält zahlreiche Namen, die zum Teil neu gelesen und ergänzt werden konnten. Es handelt sich um eine Liste der Namen der Knaben, die am lusus Troiae beteiligt waren.95

94 Nur beim Opfer für Terra Mater (Z. 224) und Apollon/Diana (Z. 230) sind die Namen von Geta, Plautianus und die Quindecimviri als Anwesende erhalten. Die Angehörigen der kaiserlichen Familie und die Quindecimviri waren aber bei allen Opfern direkt neben der Opferhandlung präsent. 